\section{Response to: 'A relationship between extracapsular involvement and response to steroid treatment in polymyalgia rheumatica: too soon to conclude?' by Yang et al}

We thank Yang et $a l^{1}$ for their comments on our whole body MRI study in patients with polymyalgia rheumatica (PMR). Our previous work in PMR revealed inflammatory changes outside the joint cavity, or 'extracapsular pattern disease', in both the shoulder and the hand. ${ }^{2}{ }^{3}$ In a preliminary analysis of four noncontrast whole-body MRIs in the present cohort, we saw an identical pattern of disease in PMR and consequently developed the scoring system described. In addition to scoring the individual sites semi-quantitatively, we also classified the overall pattern as 'extracapsular' or not, using visual pattern recognition. This Gestalt-based classification was not derived directly from any of the individual site scores.

Subsequently, after unblinding of clinical diagnosis, we derived an 'inflammation score' by summing the scores at the five sites of gadolinium enhancement which discriminated best between PMR and rheumatoid arthritis (RA) (ranking shown in supplementary table 2 in Ref. 4). This was done to compare the overall burden of imaging inflammation with circulating interleukin-6 levels in the PMR patients (supplementary figure 2 in Ref. 4). We agree with Yang et al that it is unsurprising that PMR patients with extracapsular pattern had higher scores than the other patients; statistical testing was not in fact performed for this comparison.

Yang et al enquired about the extracapsular versus intracapsular pattern in PMR versus RA. The florid extracapsular pattern we observed in PMR was also associated with some intracapsular gadolinium enhancement. The difference between PMR and RA was best seen using the pelvic sequences; here, the extracapsular sites were most distant from the synovial joints. Most early reports of musculoskeletal imaging in PMR focused on the shoulders. ${ }^{5}$ More recent 18 -fluorodeoxyglucose positron emission tomography (18-FDG-PET)/CT studies have, like ours, identified focal 18-FDG uptake at capsular/entheseal sites distant from articular joints, including the hip pericapsular tissue and sites adjacent to the pubic symphysis. ${ }^{6}$ Although all these sites of focal 18-FDG uptake were termed 'bursitis', we are concerned that this term may lead to an assumption that the synovial lining of the bursa is the primary site of inflammation in PMR. We therefore prefer the more general term 'extracapsular' to describe the PMR-related pattern of inflammation which is situated around the joint capsules. ${ }^{7}$

All of the PMR patients included in our MRI study showed a significant response to glucocorticoid therapy, supporting the clinical diagnosis. However, in informal discussions with patients, ${ }^{8}$ despite all patients with PMR having an initial good response to glucocorticoids, some patients reported a 'tail' of persistent symptoms, whereas others said they feel completely 'back to normal[for them]'. We wished to explore whether this heterogeneity in the patient experience may reflect differences in the pattern of inflammation. In our MRI study, the 'nonextracapsular' group of PMR patients had greater fatigue and functional impairment at first follow-up. It is not possible to determine whether or not this was because this group also reported slightly greater fatigue pretreatment; visual analogue scales behave non-linearly near the extremes.
We agree that further studies are required to replicate our observation that presence of this extracapsular pattern appears to be prognostically informative, at least in terms of completeness of patient-reported glucocorticoid response. In judging glucocorticoid response, we would advise researchers to continue to collect patient-relevant outcome data, ${ }^{9}$ rather than relying solely on 'objective' imaging tests. Indeed, we feel that it would be too soon to conclude from current data that MRI is a validated tool for disease activity monitoring in PMR, since our research question was not about disease monitoring but prognostic stratification.

\section{Sarah L Mackie, Dennis G McGonagle}

University of Leeds. Leeds Institute of Rheumatic and Musculoskeletal Medicine, Chapel Allerton Hospital, Leeds, UK

Correspondence to Dr Sarah L Mackie, University of Leeds.Leeds Institute of Rheumatic and Musculoskeletal Medicine, Chapel Allerton Hospital, Leeds LS7 4SA, UK; s.l.mackie@leeds.ac.uk

Twitter Follow Sarah Mackie at @Sarah_L_Mackie

Competing interests None declared.

Provenance and peer review Commissioned; internally peer reviewed.

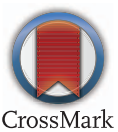

To cite Mackie SL, McGonagle DG. Ann Rheum Dis 2016;75:e17.

Received 28 December 2015

Accepted 30 December 2015

Published Online First 27 January 2016

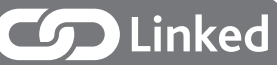

http://dx.doi.org/10.1136/annrheumdis-2015-208956

Ann Rheum Dis 2016;75:e17. doi:10.1136/annrheumdis-2015-208962

\section{REFERENCES}

1 Yang $L$, Zhou $H$, Tang $H$, et al. relationship between extracapsular involvement and response to steroid treatment in polymyalgia rheumatica: too soon to conclude? Ann Rheum Dis 2016;75:e16.

2 Marzo-Ortega $\mathrm{H}$, Rhodes LA, Tan AL, et al. Evidence for a different anatomic basis for joint disease localization in polymyalgia rheumatica in comparison with rheumatoid arthritis. Arthritis Rheum 2007;56:3496-501.

3 McGonagle D, Pease C, Marzo-Ortega $\mathrm{H}$, et al. Comparison of extracapsular changes by magnetic resonance imaging in patients with rheumatoid arthritis and polymyalgia rheumatica. J Rheumatol 2001;28:1837-41.

4 Mackie SL, Pease CT, Fukuba E, et al. Whole-body MRI of patients with polymyalgia rheumatica identifies a distinct subset with complete patient-reported response to glucocorticoids. Ann Rheum Dis 2015;74:2188-92.

5 Mackie SL, Koduri G, Hill CL, et al. Accuracy of musculoskeletal imaging for the diagnosis of polymyalgia rheumatica: systematic review. RMD Open 2015;1:e000100.

6 Rehak Z, Vasina J, Nemec P, et al. Various forms of F-FDG PET and PET/CT findings in patients with polymyalgia rheumatica. Biomedical papers of the Medical Faculty of the University Palacky, Olomouc, Czechoslovakia, 2015.

7 McGonagle D, Pease C, Marzo-Ortega $\mathrm{H}$, et al. The case for classification of polymyalgia rheumatica and remitting seronegative symmetrical synovitis with pitting edema as primarily capsular/entheseal based pathologies. J Rheumatol 2000;27:837-40

8 Mackie SL, Arat S, da Silva J, et al. Polymyalgia Rheumatica (PMR) Special Interest Group at OMERACT 11: outcomes of importance for patients with PMR. J Rheumatol 2014:41:819-23.

9 Helliwell T, Brouwer E, Pease CT, et al. Development of a provisional core domain se for polymyalgia rheumatica: report from the OMERACT 12 polymyalgia rheumatica working group. J Rheumatol 2016;43:182-6. 